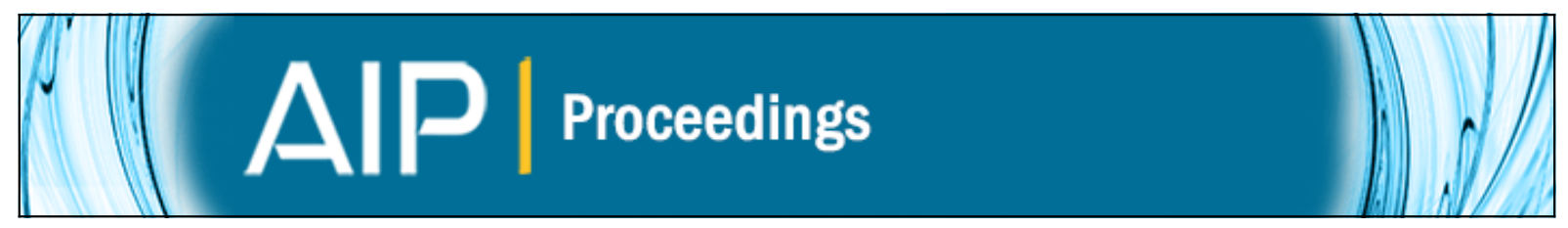

\title{
The relativistic inverse stellar structure problem
}

Lee Lindblom

Citation: AIP Conference Proceedings 1577, 153 (2014); doi: 10.1063/1.4861951

View online: http://dx.doi.org/10.1063/1.4861951

View Table of Contents: http://scitation.aip.org/content/aip/proceeding/aipcp/1577?ver=pdfcov

Published by the AIP Publishing

Articles you may be interested in

Inversion method in stellar pulsation

AIP Conf. Proc. 895, 219 (2007); 10.1063/1.2720425

An inverse method for stellar population synthesis

AIP Conf. Proc. 761, 96 (2005); 10.1063/1.1913920

Inverse variational problem and canonical structure of Burgers equations

J. Math. Phys. 46, 043506 (2005); 10.1063/1.1852700

Some inverse problems in structural acoustics

J. Acoust. Soc. Am. 105, 969 (1999); 10.1121/1.425304

Prolongation structures and a generalized inverse scattering problem

J. Math. Phys. 17, 1867 (1976); 10.1063/1.522808 


\title{
The Relativistic Inverse Stellar Structure Problem
}

\author{
Lee Lindblom \\ Theoretical Astrophysics, California Institute of Technology, Pasadena, CA 91125
}

\begin{abstract}
The observable macroscopic properties of relativistic stars (whose equations of state are known) can be predicted by solving the stellar structure equations that follow from Einstein's equation. For neutron stars, however, our knowledge of the equation of state is poor, so the direct stellar structure problem can not be solved without modeling the highest density part of the equation of state in some way. This talk will describe recent work on developing a model independent approach to determining the high-density neutron-star equation of state by solving an inverse stellar structure problem. This method uses the fact that Einstein's equation provides a deterministic relationship between the equation of state and the macroscopic observables of the stars which are composed of that material. This talk illustrates how this method will be able to determine the high-density part of the neutron-star equation of state with few percent accuracy when high quality measurements of the masses and radii of just two or three neutron stars become available. This talk will also show that this method can be used with measurements of other macroscopic observables, like the masses and tidal deformabilities, which can (in principle) be measured by gravitational wave observations of binary neutron-star mergers.
\end{abstract}

Keywords: equation of state, stars: neutron, relativity

PACS: $04.40 . \mathrm{Dg}, 97.60 . \mathrm{Jd}, 26.60 . \mathrm{Kp}, 26.60 . \mathrm{Dd}$

\section{STANDARD STELLAR STRUCTURE PROBLEM}

Models of stars in general relativity theory are constructed by solving the relativistic stellar structure equations that follow from Einstein's equation. The most widely used form of these equations, for non-rotating stellar models, was first derived by Oppenheimer and Volkoff [1]:

$$
\begin{aligned}
& \frac{d m}{d r}=4 \pi r^{2} \varepsilon, \\
& \frac{d p}{d r}=-(\varepsilon+p) \frac{m+4 \pi r^{3} p}{r(r-2 m)} .
\end{aligned}
$$

These equations determine $m(r)$ and $p(r)$, the mass of the star contained within a sphere of radius $r$ and the pressure respectively. These equations are incomplete however, and must be supplemented by giving an equation of state that characterizes the material contained within the star. In particular, the relationship between the total energy density $\varepsilon$ and the pressure $p$ must be specified. The addition of the relationship $\varepsilon=\varepsilon(p)$ completes the structure equations, (1) and (2), making them a first-order set of ordinary differential equations that can be solved using standard techniques. These equations are usually integrated starting at the center of the star, where $r=0$, using the boundary conditions $m(0)=0$, to ensure that the stellar model is non-singular, and $p(0)=p_{c}$ to select the central value of the pressure. The value of the central pressure $p_{c}$ can be chosen arbitrarily, with larger $p_{c}$ generally corresponding to stellar models having larger masses. The stellar structure equations, (1) and (2), are then integrated toward larger values of $r$ until the surface of the star at $r=R$ is reached, where $p(R)=0$. The total mass of the star is given by $M=m(R)$.

The central pressure $p_{c}$ is a freely specifiable parameter, so the stellar structure equations determine a one-parameter family of solutions, $m\left(r, p_{c}\right)$ and $p\left(r, p_{c}\right)$, for any given equation of state. In general, the macroscopic observables associated with these stellar models, like the total mass $M$ and radius $R$, also depend on this parameter $p_{c}$. Therefore the macroscopic observables associated with any particular equation of state can be thought of as a curve, parameterized by $p_{c}$, in the space of those observables. This talk will focus on the particular case of masses $M\left(p_{c}\right)$ and radii $R\left(p_{c}\right)$. The standard stellar structure problem can be thought of as an abstract map that associates an equation of state (which is in effect a curve though the space of energy densities and pressures) with a curve through the space of observables. Figure 1 illustrates what this abstract map looks like for the particular case of the mass-radius curve of observables. 


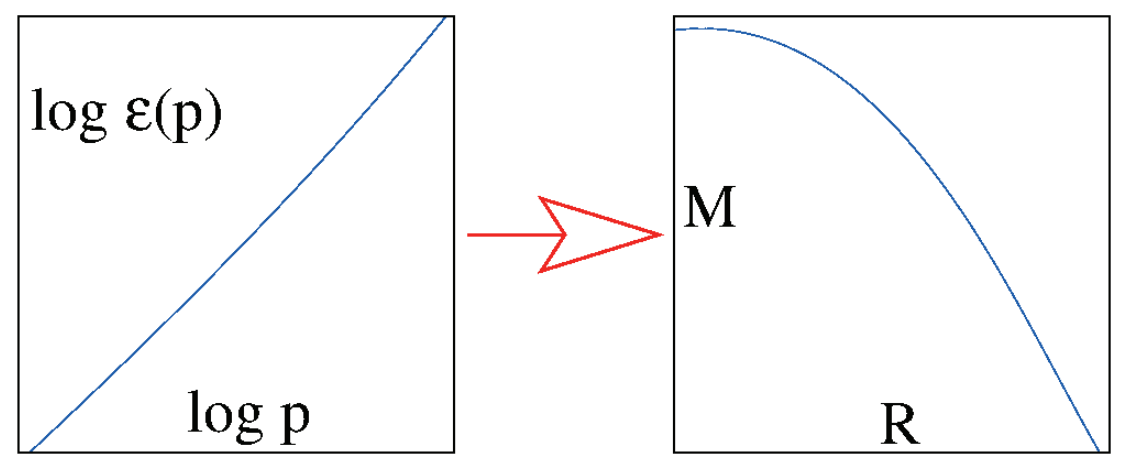

FIGURE 1. Standard relativistic stellar structure problem can be thought of as an abstract map between an equation of state (a curve in the space of densities and pressures) and a curve of macroscopic observables, like the mass-radius curve.

\section{INVERSE STELLAR STRUCTURE PROBLEM}

When the equation of state is well understood, as in white dwarf stars, the standard stellar structure problem is very useful. Detailed models of these stars can be computed, and their observable properties can be compared directly with observations. For cases where the equation of state is poorly know however, like neutron stars, the standard stellar structure problem is not so useful. Figure 2 illustrates the high-density portions of 34 theoretical neutron-star equations of state [2]. The range of pressures corresponding to nuclear density (dashed red line) predicted by these theoretical equations of state have a range that spans about an order of magnitude. This means that it is not possible to make reliable quantitative predictions about the structures of neutron stars simply by solving the standard stellar structure problem.

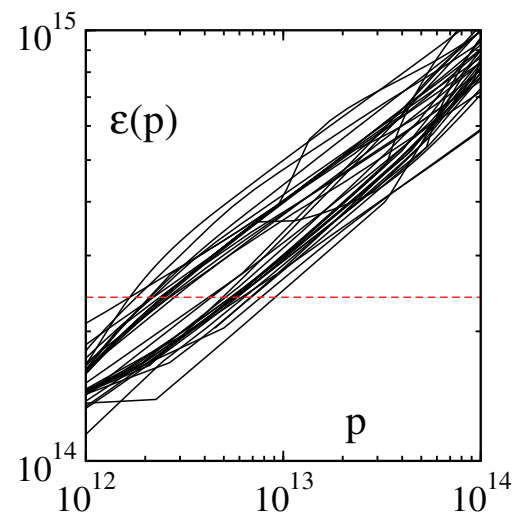

FIGURE 2. The neutron-star equation of state is poorly known, as illustrated here by the high-density portions of 34 theoretical neutron-star equations of state. The range of pressures at nuclear density (dashed red line) in these equations of state span about an order of magnitude.

The range of densities and pressures inside typical neutron stars are well beyond the range where any current or foreseeable future laboratory experiment will be able to measure the neutron-star equation of state directly. In contrast, observations of the macroscopic properties of neutron stars are becoming more numerous and more accurate all the time. What is needed then is an inverse stellar structure problem, where the equation of state is determined from a knowledge of the macroscopic observables of those stars, e.g. their masses and radii. That is, we need a way of solving the stellar structure equations for the equation of state, instead of specifying it a priori as we did as part of the definition of the standard stellar structure problem.

At an abstract level it is not hard to imagine what such an inverse stellar structure problem might look like. In Fig. 1 we visualized the standard stellar structure problem as a map that takes a curve in density-pressure space (i.e. the equation of state) and identifies it with a curve in the space of macroscopic observables. From this perspective, the inverse stellar structure problem corresponds to the inverse of this abstract map. That is, the inverse stellar structure problem is the abstract map, illustrated in Fig. 3, that takes a curve in the space of macroscopic observables and from 
it determines the corresponding curve in density-pressure space.

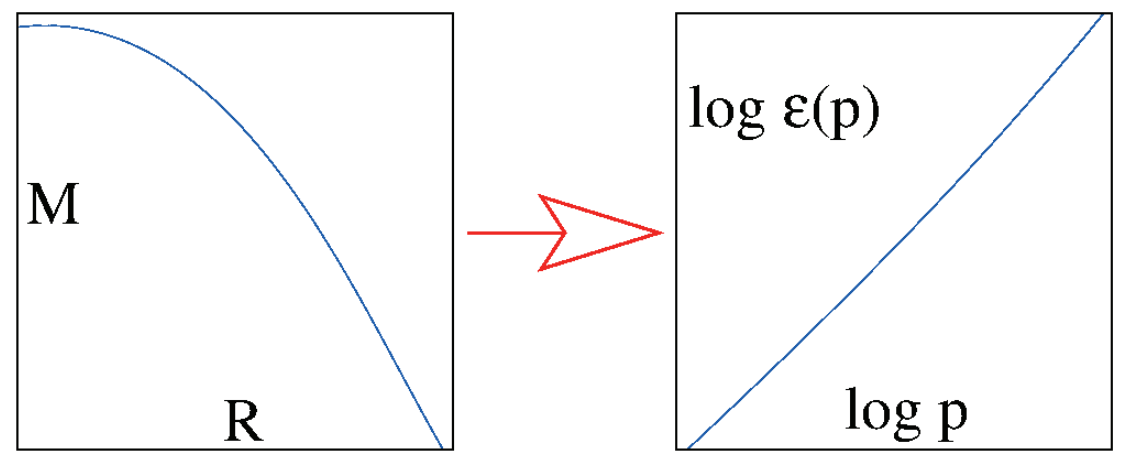

FIGURE 3. Inverse stellar structure problem can be thought of as an abstract map that takes a curve of observables, like the mass-radius curve, and from it determines the equation of state of the stellar matter.

\section{TRADITIONAL SOLUTION OF THE INVERSE STELLAR STRUCTURE PROBLEM}

Let me first discuss a particular solution to the inverse stellar structure problem, developed about 20 years ago [3], that I call the traditional solution. Later in this talk I will also discuss a newer and more sophisticated approach to solving this problem. The traditional approach, however, provides a clearer insight into the way the stellar structure equations determine the solution to this inverse problem, so I think it is worth discussing.

Let us assume that the entire mass-radius curve $\{R, M\}$ is known. Let us also assume that the lowest density part of the equation of state is known, i.e., $\varepsilon=\varepsilon(p)$ is assumed to be known for $p \leq p_{i}$ and $\varepsilon(p) \leq \varepsilon\left(p_{i}\right)=\varepsilon_{i}$. Let $\left\{R_{i}, M_{i}\right\}$ denote the point on the mass-radius curve whose central pressure is $p_{i}$. We choose another point on the mass-radius curve, $\left\{R_{i+1}, M_{i+1}\right\}$, that is "close" to the point $\left\{R_{i}, M_{i}\right\}$, but has a slightly larger central pressure. Figure 4 illustrates these assumptions. The mass-radius curve is illustrated in the first panel of Fig. 4, including the particular points $\left\{R_{i}, M_{i}\right\}$ and $\left\{R_{i+1}, M_{i+1}\right\}$. The red portion of the equation of state curve, shown in the middle panel of Fig. 4 , is also assumed to be known, including the particular point $\left\{p_{i}, \varepsilon_{i}\right\}$. The red portion of the mass-radius curve in Fig. 4 represents those stellar models whose central pressures are less than $p_{i}$. The panel on the right in Fig. 4 is a cross section that represents the structure of the stellar model $\left\{R_{i+1}, M_{i+1}\right\}$. The outer portions of this model, shaded red, are composed of low density material whose equation of state is already known. The small central core of this model, shaded blue, is composed of material whose pressure and density exceed $\left\{p_{i}, \varepsilon_{i}\right\}$.
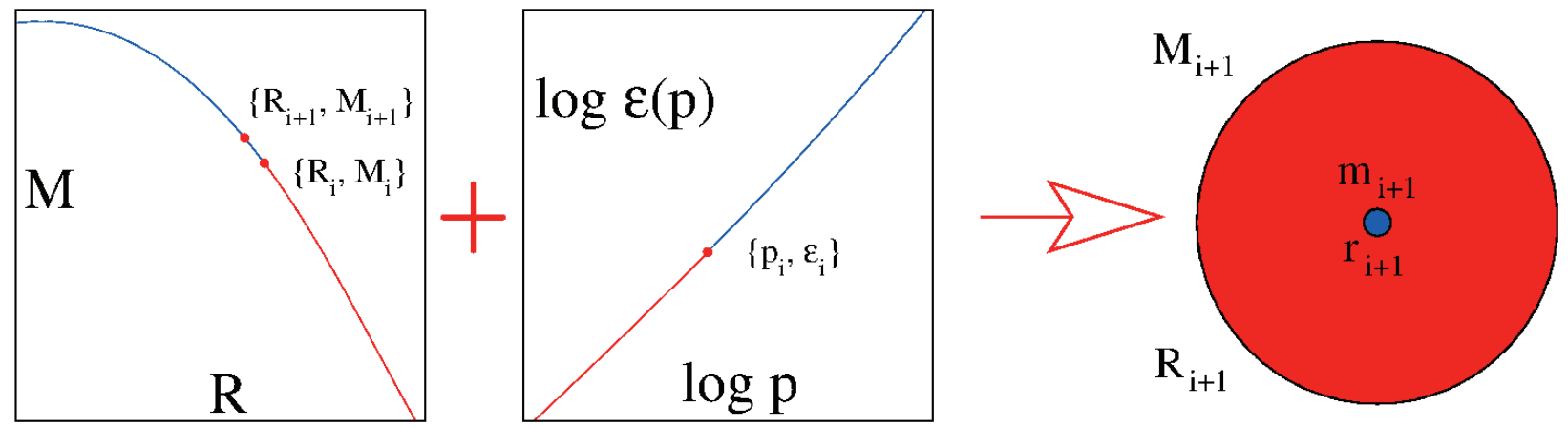

FIGURE 4. Knowledge of the complete mass-radius curve plus a knowledge of the low density equation of state determines the structure of the outer parts of the star with total mass $M_{i+1}$ and radius $R_{i+1}$. Also determined from this knowledge are the mass $m_{i+1}$ and radius $r_{i+1}$ of the small inner core of material having densities and pressures from the undetermined high-density part of the equation of state.

The relativistic stellar structure equations, (1) and (2), can be integrated to determine quantitatively the structure of the stellar model $\left\{R_{i+1}, M_{i+1}\right\}$. Generally these equations are integrated from the center of the star, $r=0$, outward. But we can not do that in this case, because we do not know the equation of state at densities contained in the core of this model. We can, however, integrate the equations starting at the surface of this star, $r=R_{i+1}$, using as initial data 
$m\left(R_{i+1}\right)=M_{i+1}$ and $p\left(R_{i+1}\right)=0$. Since the low density equation of state is known, this integration can be continued up to the point where $p=p_{i}$. The mass and radius, $\left\{r_{i+1}, m_{i+1}\right\}$, of this small high-density core can therefore be determined quantitatively from this model: $p\left(r_{i+1}\right)=p_{i}$, and $m\left(r_{i+1}\right)=m_{i+1}$.

If the point $\left\{R_{i+1}, M_{i+1}\right\}$ has been chosen to be sufficiently close to $\left\{R_{i}, M_{i}\right\}$, then the high-density core composed of material from the unknown high-density part of the equation of state will have very small mass and radius, $\left\{r_{i+1}, m_{i+1}\right\}$. In this case the structure equations, (1) and (2), can be solved as power series expansions:

$$
\begin{aligned}
m_{i+1} & =\frac{4 \pi}{3} \varepsilon_{i+1} r_{i+1}^{3}+\mathscr{O}\left(r_{i+1}^{5}\right), \\
p_{i} & =p_{i+1}-\frac{2 \pi}{3}\left(\varepsilon_{i+1}+p_{i+1}\right)\left(\varepsilon_{i+1}+3 p_{i+1}\right) r_{i+1}^{2}+\mathscr{O}\left(r_{i+1}^{4}\right) .
\end{aligned}
$$

The coefficients in these series depend on $\left\{p_{i+1}, \varepsilon_{i+1}\right\}$, the central density and pressure of the little core $\left\{r_{i+1}, m_{i+1}\right\}$. These series can therefore be inverted to determine $\left\{p_{i+1}, \varepsilon_{i+1}\right\}$ in terms of the known quantities $\left\{p_{i}, \varepsilon_{i}\right\}$ and $\left\{r_{i+1}, m_{i+1}\right\}$. This provides an additional point on the equation of state curve, as illustrated in Fig. 5. The points on the equation of state curve that are intermediate between $\left\{p_{i}, \varepsilon_{i}\right\}$ and $\left\{p_{i+1}, \varepsilon_{i+1}\right\}$ are determined by adopting a suitable interpolation formula. This procedure can be iterated to determine the equation of state for the entire range of densities that occur within the cores of neutron stars. This method has been used successfully to solve the inverse stellar structure problem using mock mass radius data constructed from a known theoretical equation of state in Lindblom [3].

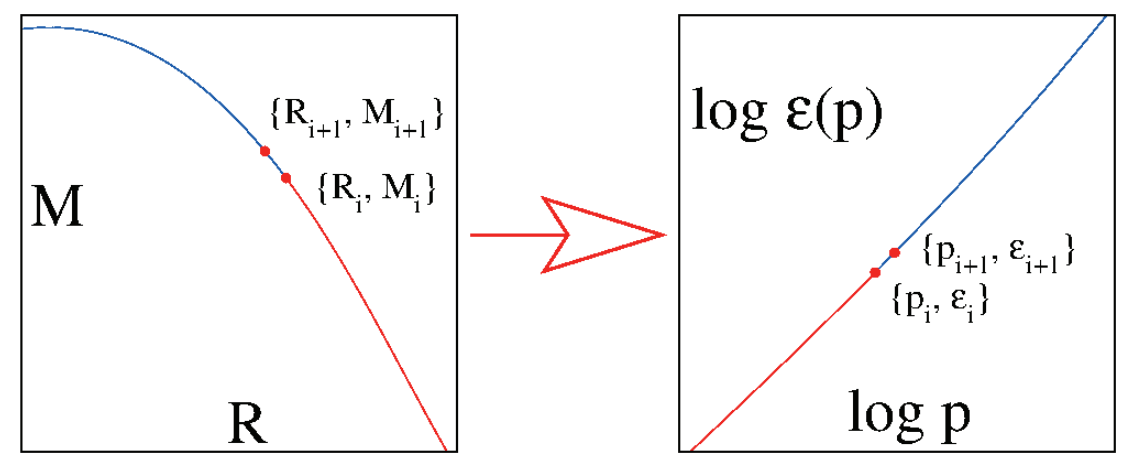

FIGURE 5. Traditional solution of the inverse stellar structure problem iteratively determines a sequence of points on the equation of state curve by inverting the power series solutions for small inner cores of material whose masses and radii are determined numerically from a knowledge of the complete mass radius curve.

This traditional method for solving the inverse stellar structure problem represents the equation of state as a table $\left\{p_{i}, \varepsilon_{i}\right\}$ for $i=1, \ldots, N_{E O S}$ plus an interpolation formula to fill in intermediate points. The accuracy of a particular implementation of this method depends on how many terms are retained in the power series expansions, (3) and (4), as well as the type of interpolation formula used. The overall accuracy of the equation of state determined in this way will also depend on the number, $N_{\text {stars }}$, of mass-radius data available, typically scaling like $N_{\text {stars }}^{-n}$ for some $n$. Since this method of representing equations of state is rather inefficient, and since this method of solving the inverse stellar structure problem converges rather slowly, many mass-radius data points would be needed to determine the equation of state with reasonable accuracy in this way. Measurements of these data are very difficult to make, so it seems unlikely that this traditional method will ever be useful for analyzing real astrophysical data.

\section{SPECTRAL APPROACH TO THE INVERSE STELLAR STRUCTURE PROBLEM}

The key idea of the spectral approach to the inverse stellar structure problem is to find a more efficient way to represent equations of state using spectral methods. Fewer observations of the macroscopic observables should then be sufficient to determine the equation of state by solving an appropriately modified version of the inverse stellar structure problem.

The basic idea is to construct efficient parametric representations of the equation of state, e.g. $\varepsilon=\varepsilon\left(p, \gamma_{k}\right)$, where the $\gamma_{k}$ are a set of parameters that fix the functional form of a particular equation of state. One example of such a representation is a simple spectral expansion of the form, $\varepsilon\left(p, \gamma_{k}\right)=\sum_{k} \gamma_{k} \Phi_{k}(p)$, where the $\Phi_{k}(p)$ are suitably chosen basis functions. Examples of such basis functions include the Fourier basis functions $\Phi_{k}(p)=e^{i k p}$, and the various 
collections of orthonormal polynomials, like the Chebyshev polynomials $\Phi_{k}(p)=T_{k}(p)$. Spectral expansions of this type are exact when an infinite number of basis functions are included in the sum. Truncating these expansions at some finite number of terms, $k \leq N_{\gamma_{k}}$, gives an approximation to the equation of state, whose errors decrease faster than any power of $N_{\gamma_{k}}$ (e.g. typically exponentially) for smooth functions. I will discuss the particular choice of parametric representation that has been useful for equations of state later in this talk.

For now, let us simply assume that we have a parametric representation, $\varepsilon=\varepsilon\left(p, \gamma_{k}\right)$, that provides reasonably accurate approximate equations of state with just a few parameters $\gamma_{k}$. We use this family of equations of state to solve the standard stellar structure equations, (1) and (2). The results include the various macroscopic observables associated with these stellar models, like their total masses $M\left(p_{c}, \gamma_{k}\right)$ and radii $R\left(p_{c}, \gamma_{k}\right)$. These model observables now depend explicitly in the parameters $\gamma_{k}$ that determine the particular equation of state, as well as the parameter $p_{c}$ that specifies the central pressure of the model. The spectral approach to the inverse stellar structure problem fixes these equation of state parameters, $\gamma_{k}$, by matching the model observables $\left\{R\left(p_{c}, \gamma_{k}\right), M\left(p_{c}, \gamma_{k}\right)\right\}$ to a collection of points $\left\{R_{i}, M_{i}\right\}$, for $i=1, \ldots, N_{\text {stars }}$, from the exact curve of macroscopic observables for these stars. The parameters $\gamma_{k}$, along with the parameters $p_{c}^{i}$ that represent the central pressures of the models whose observables are $\left\{R_{i}, M_{i}\right\}$, are determined by minimizing the quantity $\chi\left(\gamma_{k}, p_{c}^{i}\right)$, defined by,

$$
\chi^{2}\left(\gamma_{k}, p_{c}^{i}\right)=\frac{1}{N_{\text {stars }}} \sum_{i=1}^{N_{\text {stars }}}\left\{\left[\log \left(\frac{R\left(p_{c}^{i}, \gamma_{k}\right)}{R_{i}}\right)\right]^{2}+\left[\log \left(\frac{M\left(p_{c}^{i}, \gamma_{k}\right)}{M_{i}}\right)\right]^{2}\right\},
$$

with respect to each of the parameters $\gamma_{k}$ and $p_{c}^{i}$ This non-linear least squares problem can be solved using standard numerical methods. Once fixed in this way, the parameters $\gamma_{k}$ now determine an equation of state, $\varepsilon=\varepsilon\left(p, \gamma_{k}\right)$, that provides an approximate solution to the inverse stellar structure problem. The accuracies of spectral expansions typically decrease exponentially with the number of parameters $N_{\gamma_{k}}$ used in the expansion. The expectation is that the approximate equations of state constructed with this spectral approach to the inverse stellar structure problem should therefore converge rapidly to the real equation of state as the number of parameters $\gamma_{k}$ is increased.

\section{SPECTRAL REPRESENTATIONS OF NEUTRON-STAR EQUATIONS OF STATE}

The standard Oppenheimer-Volkoff representation of the stellar structure equations, (1) and (2), which use the pressure as the fundamental thermodynamic variable, is not actually the best form to use for numerical solutions (cf. Lindblom [3]). For a variety of reasons it is better to re-write those equations in terms of the relativistic enthalpy $h$, defined by

$$
h(p)=\int_{0}^{p} \frac{d p^{\prime}}{\varepsilon\left(p^{\prime}\right)+p^{\prime}},
$$

instead of the pressure $p$. I will discuss some of the benefits of the enthalpy-based stellar structure equations later in this talk. But let us focus first on the problem of finding suitable parametric enthalpy-based representations of the equation of state. The enthalpy-based equation of state consists of the pair of functions, $\varepsilon=\varepsilon(h)$ and $p=p(h)$. The simple spectral expansions of these equations would have the forms $\varepsilon\left(h, \alpha_{k}\right)=\sum_{k} \alpha_{k} \Phi_{k}(h)$ and $p\left(h, \beta_{k}\right)=\sum_{k} \beta_{k} \Phi_{k}(h)$. These simple expansions have two serious drawbacks. First, the spectral basis functions $\Phi_{k}(h)$ are typically oscillatory, while physical equations of state must be monotonically increasing functions. While it is possible to express every monotonic function using an expansion of this sort, doing so requires that the spectral coefficients satisfy a very complicated set of inequalities. The second drawback is that these expansions of $\varepsilon(h)$ and $p(h)$ require two sets of parameters, while the original $\varepsilon(p)$ expansion considered previously required only one. These simple enthalpy based expansions are therefore very inefficient, which makes them inappropriate for our purposes.

We need faithful spectral representations of the equation of state. Faithful representations in this context means ones where every choice of spectral parameters corresponds to a possible physical equation of state, and conversely ones where every physical equation of state can be represented by some choice of spectral parameters (cf. Lindblom [4]). The simple spectral expansions proposed above are not faithful. It is not possible to represent the space of all monotonically increasing functions as all possible linear combinations of any choice of basis functions, because monotonic functions do not form a vector space. Faithful spectral expansions can, however, be constructed for the 
adiabatic index, $\Gamma(h)$, defined as

$$
\Gamma(h)=\frac{\varepsilon+p}{p} \frac{d p}{d \varepsilon}=\exp \left[\sum_{k} \gamma_{k} \Phi_{k}(h)\right] .
$$

Monotonicity of the equation of state implies that the adiabatic index is positive, $\Gamma(h)>0$, but there is no requirement that it must also be monotonic. Any spectral expansion of the form given in equation (7) is therefore faithful.

Unfortunately, the adiabatic index $\Gamma(h)$ does not appear in the stellar structure equations, while $\varepsilon(h)$ and $p(h)$ do. Fortunately, however, the adiabatic index $\Gamma(h)$ determines both $\varepsilon(h)$ and $p(h)$. The definitions of the enthalpy in equation (6), and the adiabatic index in equation (7), can be written as the following pair of ordinary differential equations for $\varepsilon(h)$ and $p(h)$ :

$$
\begin{aligned}
& \frac{d p}{d h}=\varepsilon+p, \\
& \frac{d \varepsilon}{d h}=\frac{(\varepsilon+p)^{2}}{p \Gamma(h)} .
\end{aligned}
$$

Given an adiabatic index, $\Gamma(h)$, this first-order system of equations can be integrated exactly (cf. Lindblom [4]) to determine both $p(h)$ and $\varepsilon(h)$ :

$$
\begin{aligned}
p(h) & =p_{0} \exp \left[\int_{h_{0}}^{h} \frac{e^{h^{\prime}} d h^{\prime}}{\mu\left(h^{\prime}\right)}\right], \\
\varepsilon(h) & =p(h) \frac{e^{h}-\mu(h)}{\mu(h)}, \\
\mu(h) & =\frac{p_{0} e^{h_{0}}}{\varepsilon_{0}+p_{0}}+\int_{h_{0}}^{h} \frac{\Gamma\left(h^{\prime}\right)-1}{\Gamma\left(h^{\prime}\right)} e^{h^{\prime}} d h^{\prime},
\end{aligned}
$$

where $h_{0}$ is the lower bound on the range of enthalpies over which the spectral expansion is performed, $\varepsilon_{0}=\varepsilon\left(h_{0}\right)$, and $p_{0}=p\left(h_{0}\right)$. While the integrals that appear in equations (10)-(12) can not generally be done analytically, these integrals can nevertheless be done numerically very efficiently. I have found that they can be evaluated numerically with double precision accuracy using Gaussian quadrature using only ten or twelve collocation points.

All the equations of state, $\varepsilon\left(h, \gamma_{k}\right)$ and $p\left(h, \gamma_{k}\right)$, defined by equations (10)-(12), using the adiabatic index expansion given in equation (7) are faithful for any choice of basis functions. To determine whether expansions of this type are efficient and practical, I have used them to construct spectral representations of 34 theoretical neutron-star equations of state. These theoretical equation of state models are cataloged in Read, et al. [2], and references are given there to the original papers on which they are based. These theoretical equations of state are given as tables $\left\{p_{i}, \varepsilon_{i}\right\}$ for $i=1, \ldots, N_{E O S}$, from which a set of corresponding $h_{i}$ can be evaluated using equation (6), once a suitable interpolation formula is adopted. These data were fit to these parametric equations of state, $\varepsilon=\varepsilon\left(h, \gamma_{k}\right)$ and $p=p\left(h, \gamma_{k}\right)$, by minimizing the quantity $\Delta_{N_{\gamma_{k}}}^{E O S}$, defined by

$$
\left(\Delta_{N_{\gamma_{k}}}^{E O S}\right)^{2}=\frac{1}{N_{E O S}} \sum_{i=1}^{N_{E O S}}\left\{\log \left[\frac{\varepsilon\left(h_{i}, \gamma_{k}\right)}{\varepsilon_{i}}\right]\right\}^{2},
$$

with respect to each of the spectral parameters $\gamma_{k}$. Such fits were performed using several choices for the spectral basis functions $\Phi_{k}(h)$. The following simple choice was found to be quite effective for a wide range of these theoretical equations of state,

$$
\Gamma(h)=\exp \left\{\sum_{k=0}^{N_{\gamma_{k}}-1} \gamma_{k}\left[\log \left(\frac{h}{h_{0}}\right)\right]^{k}\right\} .
$$

This simple power-law expansion has therefore been adopted as the standard to use for enthalpy-based representations of the equation of state (cf. Lindblom [4]). The results of these fits are summarized in Table 1. These results show that only two or three spectral coefficients $\gamma_{k}$ are needed to produce representations of these 34 theoretical neutron-star 
TABLE 1. Summaries of the average accuracies of the direct spectral fits to 34 theoretical equations of state. The examples included in this table are the best case, PAL6, a typical average case, MS1, and the worst case, BGN1H1. Also listed are the average errors for the spectral fits to all 34 theoretical equations of state.

\begin{tabular}{l|cccc}
\hline EOS & $\Delta_{2}^{E O S}$ & $\Delta_{3}^{E O S}$ & $\Delta_{4}^{E O S}$ & $\Delta_{5}^{E O S}$ \\
\hline PAL6 & 0.0032 & 0.0016 & 0.0005 & 0.0002 \\
MS1 & 0.0277 & 0.0055 & 0.0035 & 0.0003 \\
BGN1H1 & 0.0868 & 0.0495 & 0.0439 & 0.0403 \\
\hline Average & 0.0323 & 0.0166 & 0.0124 & 0.0089 \\
\hline
\end{tabular}

equations of state with average errors of only a few percent errors over the entire range of densities that occur in the cores of neutron stars. Figure 6 illustrates in more detail the errors in these spectral equations of state for three particular cases: PAL6, MS1 and BGN1H1. These three represent the equations of state having the best (PAL6), a typical average (MS1), and the worst (BGN1H1) spectral representations. These results show that even the worst case, the BGN1H1 equation of state which has a strong phase transition within the neutron-star density range, can be represented fairly accurately with a spectral expansion having only a few spectral coefficients.
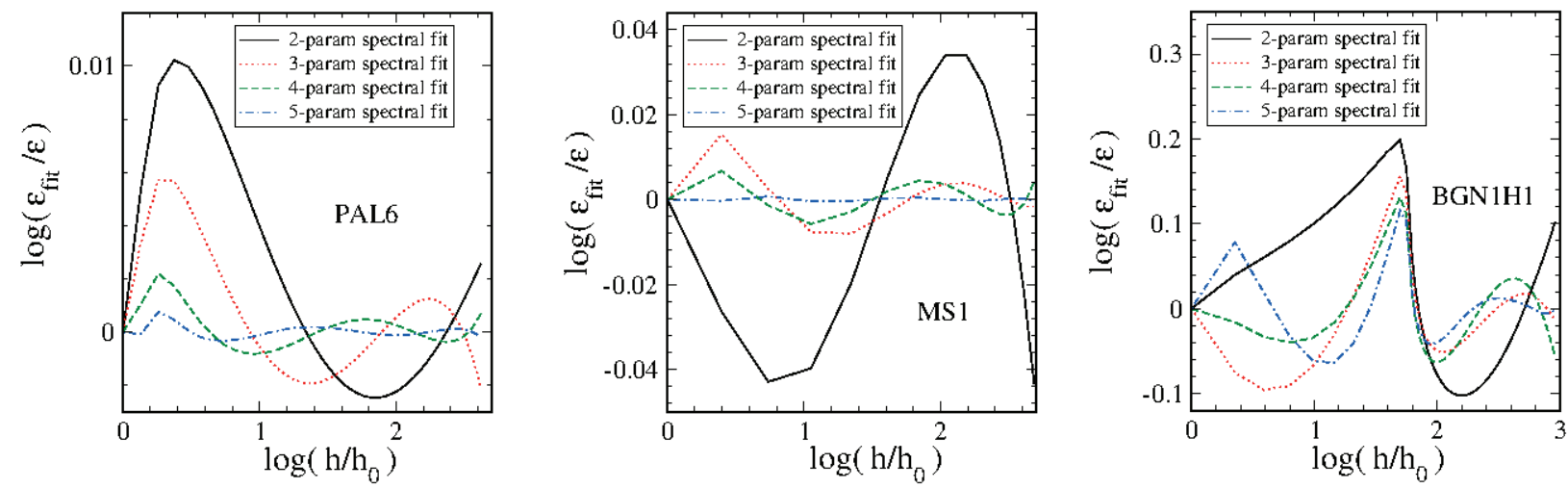

FIGURE 6. Accuracies of the spectral representations of three of the theoretical neutron-star equations of state. These three cases represent the equations of state with spectral representations having the best (PAL6), the average (MS1), and the worst (BGN1H1) accuracies.

\section{SPECTRAL SOLUTION OF THE INVERSE STELLAR STRUCTURE PROBLEM}

The standard Oppenheimer-Volkoff version of the relativistic stellar structure equations, (1) and (2), are not ideal for numerical work. The problem is that the right side of the pressure equation vanishes as the surface of the star is approached. The leading order term in the solution for the pressure $p(r)$ near the surface goes like $p \propto(R-r)^{\Gamma_{0} /\left(\Gamma_{0}-1\right)}$, where $\Gamma_{0}$ is the surface value of the adiabatic index. Consequently it is very difficult to solve $p(R)=0$ numerically to determine the location of the surface of the star. This problem can be avoided by switching to the relativistic enthalpy, $h$ defined in equation (6), as the fundamental thermodynamic variable. In this case the structure equation (2) becomes

$$
\frac{d h}{d r}=\frac{1}{\varepsilon+p} \frac{d p}{d r}=-\frac{m+4 \pi r^{3} p(h)}{r(r-2 m)} .
$$

This equation implies that the leading order term in the solution for the enthalpy $h(r)$ goes like $h \propto(R-r)$ near the surface. Consequently it is much easier numerically to solve $h(R)=0$ to determine the location of the surface of the star in this case.

Equation (14) implies that the enthalpy $h(r)$ is a monotonically decreasing function in any star. It is possible, therefore to transform the relativistic structure equations one more time by using $h$ instead of $r$ as the independent 
variable:

$$
\begin{aligned}
\frac{d r}{d h} & =-\frac{r(r-2 m)}{m+4 \pi r^{3} p(h)}, \\
\frac{d m}{d h} & =-\frac{4 \pi \varepsilon(h) r^{3}(r-2 m)}{m+4 \pi r^{3} p(h)} .
\end{aligned}
$$

The boundary conditions for these equations at the center of the star where $h=h_{c}$ are $r\left(h_{c}\right)=m\left(h_{c}\right)=0$. The solution to these equations is a relativistic stellar model with the mass $m(h)$ and radius $r(h)$ expressed as functions of the enthalpy. This version of the equations has several advantages for numerical work. First, the range of the independent variable $h_{c} \geq h \geq 0$ is fixed before the integration begins. Therefore, it is not necessary to hunt for the location of the surface by trying to solve $h(R)=0$ on the fly as part of the solution process. Second, the surface values of the total mass $M$ and radius $R$ of the model are determined simply by evaluating the solution at the endpoint of the integration where $h=0: M=m(0)$ and $R=r(0)$. The disadvantage to this approach is that the standard representation of the equation of state, $\varepsilon=\varepsilon(p)$ must be transformed into a pair of functions $\varepsilon=\varepsilon(h)$ and $p=p(h)$. This can be done in a straightforward way numerically, using the definition of the enthalpy in equation (6). In practice this disadvantage is not a real problem.

These enthalpy based representations of the stellar structure equations, (15) and (16), along with the enthalpy based spectral representations of the equation of state, equations (10)-(13), can be solved to evaluate the macroscopic observables $M\left(h_{c}, \gamma_{k}\right)$ and $R\left(h_{c}, \gamma_{k}\right)$ accurately and efficiently. These observables depend on the central value of the enthalpy $h_{c}$ in these models (in much the same way as the standard representations depend on the central pressure $p_{c}$ ), along with the spectral parameters $\gamma_{k}$ that specify a particular equation of state.

My collaborator Nathaniel Indik and I have used these techniques to construct solutions to the relativistic inverse stellar structure problem $[5,6]$. We compute the spectral coefficients $\gamma_{k}$ that determine the approximate solution to the inverse stellar structure problem by minimizing the quantity $\chi\left(h_{c}^{i}, \gamma_{k}\right)$ that measures the differences between the model values of the observables $R\left(h_{c}^{i}, \gamma_{k}\right)$ and $M\left(h_{c}^{i}, \gamma_{k}\right)$ and the data points $\left\{M_{i}, R_{i}\right\}$ taken from the exact curve of observables:

$$
\chi^{2}\left(h_{c}^{i}, \gamma_{k}\right)=\frac{1}{N_{\text {stars }}} \sum_{i=1}^{N_{\text {stars }}}\left\{\left[\log \left(\frac{M\left(h_{c}^{i}, \gamma_{k}\right)}{M_{i}}\right)\right]^{2}+\left[\log \left(\frac{R\left(h_{c}^{i}, \gamma_{k}\right)}{R_{i}}\right)\right]^{2}\right\} .
$$

The quantity $\chi\left(h_{c}^{i}, \gamma_{k}\right)$ is minimized with respect to variations in the $\gamma_{k}$, as well as the parameters $h_{c}^{i}$ that specify the central values of the enthalpies in the stars with observables $\left\{R_{i}, M_{i}\right\}$. This minimization is accomplished using standard numerical methods.

We have tested this method by constructing sets of mock observables $\left\{R_{i}, M_{i}\right\}$ based on the 34 theoretical neutron star equations of state described earlier. For each equation of state, we select $2 \leq N_{\text {stars }} \leq 5$ pairs of observables. The masses of these mock data are chosen to be uniformly spaced between $1.2 M_{\odot}$ (a typical minimum astrophysical neutron-star mass), and the maximum-mass model associated with that particular equation of state. Figure 7 illustrates these mock data points for the $N_{\text {stars }}=5$ cases of the PAL6, the MS1 and the BGN1H1 equations of state.

We then solve the inverse stellar structure problem by solving for the parameters $\gamma_{k}$ and $h_{c}^{i}$ that minimize $\chi\left(h_{c}^{i}, \gamma_{k}\right)$ for the data sets consisting of $N_{\text {stars }}$ observable data pairs from each of the 34 theoretical equations of state. We tested the accuracy of the equations of state determined in this way by comparing the resulting spectral model equation of state $\varepsilon\left(h, \gamma_{k}\right)$ with the tabulated exact theoretical equation of state $\left\{p_{i}, \varepsilon_{i}, h_{i}\right\}$ that was used to construct the mock data. We evaluate the differences between these using the average error measure $\Delta_{N_{\gamma_{k}}}^{M R}$ defined by

$$
\left(\Delta_{N_{\gamma_{k}}}^{M R}\right)^{2}=\frac{1}{N_{\mathrm{EOS}}} \sum_{i=1}^{N_{\mathrm{EOS}}}\left\{\log \left[\frac{\varepsilon\left(h_{i}, \gamma_{k}\right)}{\varepsilon_{i}}\right]\right\}^{2}
$$

These results are summarized in Table 2. The average errors in the spectral equations of state determined by solving the inverse stellar structure problem $\Delta_{N_{\gamma_{k}}}^{M R}$ are given in the left columns of Table 2. These error values, $\Delta_{N_{\gamma_{k}}}^{M R}$, can be compared to those constructed earlier using direct spectral fits to the equation of state tables, $\Delta_{N_{\gamma_{k}}}^{E O S}$, which are reproduced in the right columns of Table 2. We see that the accuracies of the equations of state determined by solving the inverse stellar structure problem using spectral methods are somewhat less accurate than the optimal spectral fits to those equations of state. This is not surprising. For the smoothest equations of state however, like PAL6, the 


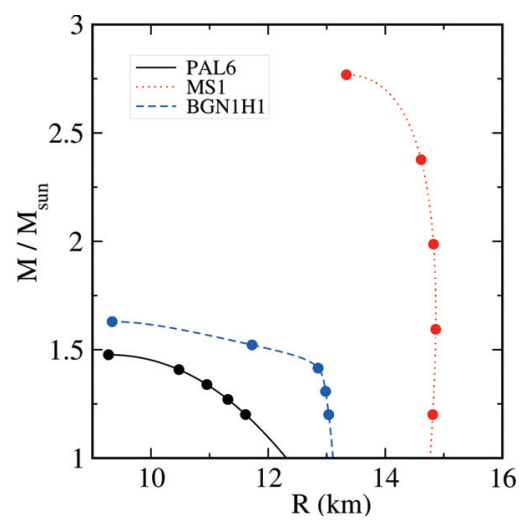

FIGURE 7. Mock data for the masses and radii $\left\{R_{i}, M_{i}\right\}$ were selected from the mass-radius curves of each of the 34 theoretical neutron-star equations of state. Shown here are the mass-radius curves for the PAL6, MS1 and the BGN1H1 equations of state with the $N_{\text {stars }}=5$ data points selected from each curve. Mock data were chosen to be equally spaced in mass between $1.2 M_{\odot}$ (a typical minimum astrophysical neutron-star mass) and the maximum mass model for each particular equation of state.

difference between the inverse stellar structure equation of state and the ones determined by doing direct spectral fits are negligible. Even for equations of state having sharp phase transitions, like BGN1H1, the errors in the inverse stellar structure equations of state are only a few times worse than the best fits.

TABLE 2. Accuracies of the equations of state determined by the spectral approach to the inverse stellar structure problem based on mass-radius data. The examples included in this table are the best case, PAL6, a typical median case, MS1, and the worst case, BGN1H1. Also listed are the average errors for all 34 theoretical equations of state. The accuracies from Table 1 of the direct spectral fits to these equations of state are also listed in the right columns of this table for comparison.

\begin{tabular}{l|cccc|cccc}
\hline EOS & $\Delta_{2}^{M R}$ & $\Delta_{3}^{M R}$ & $\Delta_{4}^{M R}$ & $\Delta_{5}^{M R}$ & $\Delta_{2}^{E O S}$ & $\Delta_{3}^{E O S}$ & $\Delta_{4}^{E O S}$ & $\Delta_{5}^{E O S}$ \\
\hline PAL6 & 0.0034 & 0.0018 & 0.0007 & 0.0003 & 0.0032 & 0.0016 & 0.0005 & 0.0002 \\
MS1 & 0.0474 & 0.0157 & 0.0132 & 0.0009 & 0.0277 & 0.0055 & 0.0035 & 0.0003 \\
BGN1H1 & 0.1352 & 0.1702 & 0.1356 & 0.1382 & 0.0868 & 0.0495 & 0.0439 & 0.0403 \\
\hline Average & 0.0396 & 0.0289 & 0.0276 & 0.0239 & 0.0323 & 0.0166 & 0.0124 & 0.0089 \\
\hline
\end{tabular}

The overall accuracies of the equations of state determined by solving the spectral version of the inverse stellar structure problem are quite impressive. These equations of state can be determined using spectral methods with average errors of just a few percent over the entire range of neutron star densities, using (high quality) measurements of the masses and radii of just two or three neutron stars. Even the worst case, an equation of state with a sharp phase transition in the neutron-star density range, is determined with accuracy levels around 15\% using high quality measurements of just a few masses and radii. Figure 8 illustrates in more detail the errors in the equations of state obtained with mock mass-radius data from the three equations of state PAL6, MS1 and BGN1H1. These error graphs are qualitatively similar to those displayed in Fig. 6 for the direct spectral fits.

\section{INVERSE STELLAR STRUCTURE PROBLEM USING TIDAL DEFORMABILITIES}

The discussion of the inverse stellar structure problem in this talk has focused up to this point on developing a nuclearphysics model independent method for using mass-radius data to determine the equation of state of neutron star matter. The advances in observational methods over the past few years has dramatically increased the number and quality of the observations of neutron star masses and radii. It seems likely that these improvements will continue, and that these observations will eventually provide high enough quality data to determine the equation of state with good accuracy. Other types of observations can provide measurements of other neutron-star observables, and in many cases these observables can also be used to solve the inverse stellar structure problem. In particular Flannigan and Hinderer [7] have pointed out (and this has been confirmed by a number of others, e.g., Lackey, et al., $[8,9]$ ) that the masses and tidal deformabilities of neutron stars could be measured by observing the gravitational waveforms produced by the 

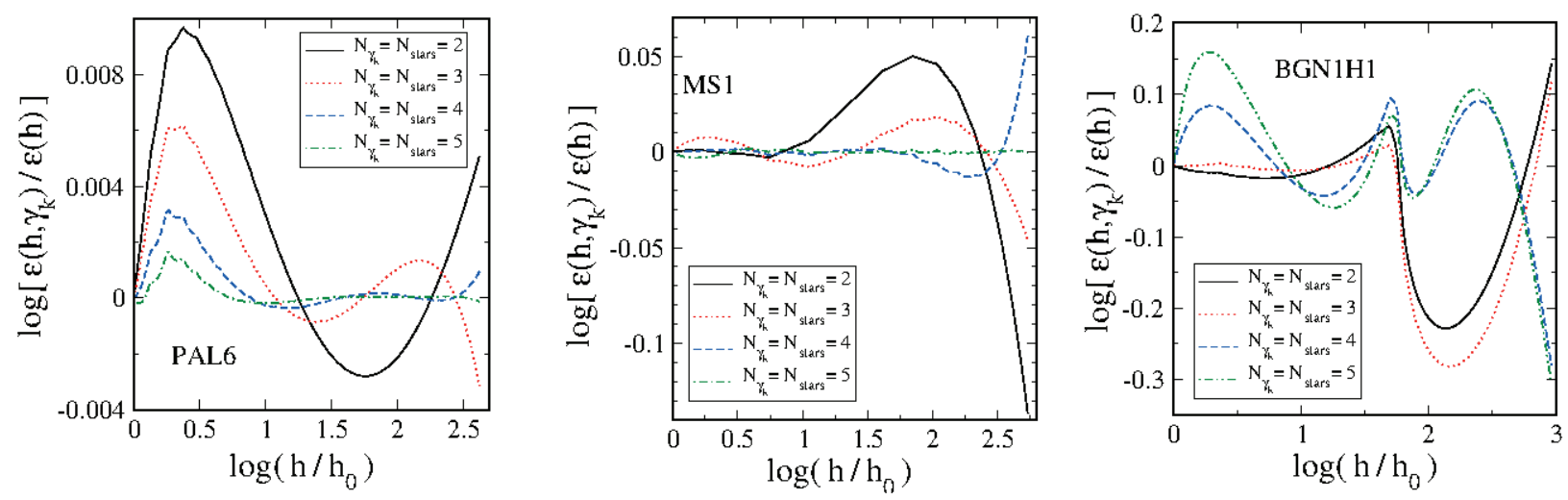

FIGURE 8. Accuracies of the spectral representations determined by solving the inverse stellar structure problem using mock mass-radius data from three of the theoretical neutron-star equations of state. These three cases represent the equations of state whose spectral representations have the best (PAL6), the average (MS1), and the worst (BGN1H1) accuracies.

inspiral and mergers of neutron-star binary systems. In this last part of my talk I will describe some recent work (done in collaboration with Nathaniel Indik) that tests how well the spectral approach to the inverse stellar structure problem works when applied to (ideal) mass and tidal deformability data.

The tidal deformability, $\Lambda$, measures the amount by which a star distorts it shape in response to the application of a tidal force from a nearby star (or black hole). The tidal deformability depends on the equation of state of the star, and therefore its measurement can in principle be used to determine the equation of state of the stellar matter. For a given equation of state, the tidal deformability can be calculated for model stars using methods first developed by Hinderer [10,11], and then transformed into the somewhat more efficient method described here by Lindblom and Indik [6]. The stellar structure equations, (15) and (16), are solved for $m(h)$ and $r(h)$, together with a third equation that determines a function $y(h)$ which describes the time independent quadrapole deformations of a relativistic star,

$$
\frac{d y}{d h}=\frac{(r-2 m)(y+1) y}{m+4 \pi r^{3} p}+y+\frac{\left(m-4 \pi r^{3} \varepsilon\right) y}{m+4 \pi r^{3} p}+\frac{4 \pi r^{3}(5 \varepsilon+9 p)-6 r}{m+4 \pi r^{3} p}+\frac{4 \pi r^{3}(\varepsilon+p)^{2}}{p \Gamma\left(m+4 \pi r^{3} p\right)}-\frac{4\left(m+4 \pi r^{3} p\right)}{r-2 m} .
$$

The appropriate initial condition for $y(h)$ at the center of the star, $h=h_{c}$, is $y\left(h_{c}\right)=2$. After this integration, the values of the resulting functions are evaluated at the surface of the star, $h=0$, to determine the quantities $M=m(0), R=r(0)$, and $Y=y(0)$. The (dimensionless) tidal deformability $\Lambda$ is then determined by the expression,

$$
\Lambda(C, Y)=\frac{16}{15 \Xi}(1-2 C)^{2}[2+2 C(Y-1)-Y],
$$

where $C=M / R$, and $\Xi$ is given by

$$
\begin{aligned}
\Xi(C, Y)= & 4 C^{3}\left[13-11 Y+C(3 Y-2)+2 C^{2}(1+Y)\right]+3(1-2 C)^{2}[2-Y+2 C(Y-1)] \log (1-2 C) \\
& +2 C[6-3 Y+3 C(5 Y-8)] .
\end{aligned}
$$

For a given equation of state, the mass $M\left(h_{c}, \gamma_{k}\right)$ and tidal deformability $\Lambda\left(h_{c}, \gamma_{k}\right)$ of a stellar model will depend on the central value of the enthalpy $h_{c}$ as well as the parameters $\gamma_{k}$ that characterized the equation of state. Using the same spectral approach to the inverse stellar structure problem described earlier, these parameters can be fixed by matching the model observables, $\left\{\Lambda\left(h_{c}^{i}, \gamma_{k}\right), M\left(h_{c}^{i}, \gamma_{k}\right)\right\}$, with data $\left\{\Lambda_{i}, M_{i}\right\}$ from the exact curve of masses and tidal deformabilities. As before, this matching is done by minimizing the quantity, $\chi\left(h_{c}^{i}, \gamma_{k}\right)$, defined by

$$
\chi^{2}\left(h_{c}^{i}, \gamma_{k}\right)=\frac{1}{N_{\text {stars }}} \sum_{i=1}^{N_{\text {stars }}}\left\{\left[\log \left(\frac{M\left(h_{c}^{i}, \gamma_{k}\right)}{M_{i}}\right)\right]^{2}+\left[\log \left(\frac{\Lambda\left(h_{c}^{i}, \gamma_{k}\right)}{\Lambda_{i}}\right)\right]^{2}\right\},
$$

with respect to variations in the parameters $h_{c}^{i}$ and $\gamma_{k}$. The resulting values of $\gamma_{k}$ determine an equation of state that serves as an approximate solution to the inverse stellar structure problem. The results of our solutions to this problem 
are described in Table 3 for mock $\left\{\Lambda_{i}, M_{i}\right\}$ data computed with the 34 theoretical neutron-star equations of state described earlier. The accuracies of these solutions are determined by evaluating the equation of state error measures $\Delta_{N_{\gamma_{k}}}^{M \Lambda}$, defined by

$$
\left(\Delta_{N_{\gamma_{k}}}^{M \Lambda}\right)^{2}=\frac{1}{N_{\mathrm{EOS}}} \sum_{i=1}^{N_{\mathrm{EOS}}}\left\{\log \left[\frac{\varepsilon\left(h_{i}, \gamma_{k}\right)}{\varepsilon_{i}}\right]\right\}^{2}
$$

Also listed in Table 3 are the analogous error measures $\Delta_{N_{\gamma_{k}}}^{M R}$ for the equations of state determined by solving the inverse stellar structure problem using mass and radius data instead of mass and tidal deformabilities. These results show that mass and tidal deformability data is about as effective as mass and radius data in determining the equation of state.

TABLE 3. Accuracies of the equations of state determined by the spectral approach to the inverse stellar structure problem based on mass-tidal deformability data. The examples included in this table are the best case, PAL6, a typical median case, MS1, and the worst case, BGN1H1. Also listed are the average errors for all 34 theoretical equations of state. The accuracies from Table 2 of the solutions based on mass-radius data are also listed in the right columns of this table for comparison.

\begin{tabular}{l|cccc|cccc}
\hline EOS & $\Delta_{2}^{M \Lambda}$ & $\Delta_{3}^{M \Lambda}$ & $\Delta_{4}^{M \Lambda}$ & $\Delta_{5}^{M \Lambda}$ & $\Delta_{2}^{M R}$ & $\Delta_{3}^{M R}$ & $\Delta_{4}^{M R}$ & $\Delta_{5}^{M R}$ \\
\hline PAL6 & 0.0034 & 0.0019 & 0.0008 & 0.0003 & 0.0034 & 0.0018 & 0.0007 & 0.0003 \\
MS1 & 0.0465 & 0.0141 & 0.0129 & 0.0008 & 0.0474 & 0.0157 & 0.0132 & 0.0009 \\
BGN1H1 & 0.1356 & 0.1652 & 0.1445 & 0.1363 & 0.1352 & 0.1702 & 0.1356 & 0.1382 \\
\hline Average & 0.0403 & 0.0295 & 0.0304 & 0.0291 & 0.0396 & 0.0289 & 0.0276 & 0.0239 \\
\hline
\end{tabular}

\section{SUMMARY}

This talk has discussed various aspects of the relativistic inverse stellar structure problem, i.e. the problem of determining the equation of state of the stellar matter from a knowledge of a curve through the space of macroscopic observables of those stars. Some recent work on the use of spectral methods was described. Spectral methods are a very efficient way to represent functions, and the particular spectral expansions described here were shown to be capable of describing a wide variety of theoretical neutron-star equations of state with accuracies of a few percent using spectral approximations with only two or three spectral parameters. It was also shown that these spectral parameters could be determined by fitting models of the masses and radii or the masses and tidal deformabilities of neutron stars with accuracies that are only somewhat less accurate that the optimal spectral fits to those equations of state.

The spectral solutions to the inverse stellar structure problem described here were based on the use of very high quality measurements of the macroscopic observables that are uniformly distributed across the astrophysically relevant range of neutron-star masses. It remains to be seen how well these spectral methods perform when more realistic data us used, i.e. data with finite measurement errors and data that is maldistributed in some way. I plan to investigate those issues at some point in the near future.

\section{ACKNOWLEDGMENTS}

I thank the organizers of the Fifth Leopoldo García-Colín Mexican Meeting on Mathematical and Experimental Physics for arranging a scientifically interesting and extremely enjoyable conference. I am grateful for having had the opportunity to participate, to renew old scientific friendships, and to make a number of new ones. I also thank the Mathematical Sciences Center at Tsinghua University in Beijing, China for their hospitality during the time this manuscript was being written. The research reported in this talk was supported by a grant from the Sherman Fairchild Foundation and by NSF grants PHY1005655 and DMS1065438. 


\section{REFERENCES}

1. J. R. Oppenheimer, and G. M. Volkoff, Physical Review 55, 374 (1939).

2. J. S. Read, B. D. Lackey, B. J. Owen, and J. L. Friedman, Phys. Rev. D79, 124032 (2009).

3. L. Lindblom, Astrophys. J. 398, 569-573 (1992).

4. L. Lindblom, Phys. Rev. D 82, 103011 (2010).

5. L. Lindblom, and N. M. Indik, Phys. Rev. D 86, 084003 (2012).

6. L. Lindblom, and N. M. Indik, Phys. Rev. D (2013), (submitted), arXiv:1310.0803.

7. T. Hinderer, and E. Flannigan, Phys. Rev. D 77, 021502 (2008).

8. B. D. Lackey, K. Kyutoku, M. Shibata, P. R. Brady, and J. L. Friedman, Phys. Rev. D 85, 044061 (2012).

9. B. D. Lackey, K. Kyutoku, M. Shibata, P. R. Brady, and J. L. Friedman, Phys. Rev. D (2013), arXiv:1303.6298.

10. T. Hinderer, Astrophys. J. 677, 1216-1220 (2008).

11. T. Hinderer, Astrophys. J. 697, 964 (2009). 